\title{
The Future of the Time Domain with LSST
}

\author{
Lucianne M. Walkowicz \\ Department of Astrophysical Sciences, Princeton University, Princeton, NJ 08544, USA \\ email: lucianne@astro.princeton.edu \\ Invited Talk
}

\begin{abstract}
Summary. In the coming decade LSST's combination of all-sky coverage, consistent long-term monitoring and flexible criteria for event identification will revolutionize studies of a wide variety of astrophysical phenomena. Time-domain science with LSST encompasses objects both familiar and exotic, from classical variables within our Galaxy to explosive cosmological events. Increased sample sizes of known-but-rare observational phenomena will quantify their distributions for the first time, thus challenging existing theories. Perhaps most excitingly, LSST will provide the opportunity to sample previously untouched regions of parameter space. LSST will generate 'alerts' within 60 seconds of detecting a new transient, permitting the community to follow up unusual events in greater detail. However, follow-up will remain a challenge as the volume of transients will easily saturate available spectroscopic resources. Characterization of events and access to appropriate ancillary data (e.g. from prior observations, either in the optical or in other passbands) will be of the utmost importance in prioritizing follow-up observations. The incredible scientific opportunities and unique challenges afforded by LSST demand organization, forethought and creativity from the astronomical community. To learn more about the telescope specifics and survey design, as well as obtaining a overview of the variety of the scientific investigations that LSST will enable, readers are encouraged to look at the LSST Science Book: http://www.Isst.org/lsst/scibook. Organizational details of the LSST science collaborations and management may be found at http://www.lsstcorp.org.
\end{abstract}

\section{Optimal Strategies for Transient Surveys with Wide-Field Radio Telescopes}

\author{
J.-P. Macquart, N. Clarke, P. Hall and T. Colegate \\ ICRAR, Curtin University, Bentley, WA 6102, Australia \\ email: jean-pierre.macquart@icrar.org
}

Summary. Fast-time-scale radio transients open new vistas on the physics of high brightnesstemperature emission, extreme states of matter and the physics of strong gravitational fields. The next generation of wide-field, highly-sensitive large- $\mathrm{N}$ radio arrays such as LOFAR, ASKAP, the MWA and the SKA offer access to an enormous volume of new transients' parameter space. This talk described how we develop a formalism to investigate the optimal means of exploiting those facilities in terms of two metrics (event detection rate, and observed detection rate per cost of hardware and operations), and how we employ that framework to assess the relative merits of various antenna configurations and backend architectures. It discussed how we compare fly's-eye survey modes against incoherent and coherent combination of the interferometric signal, and concluded by examining, in the context of SKA-scale aperture arrays, backend and beamforming configurations that maximise scientific return for a given hardware cost. 